\title{
Epigenetic signature predicts overall survival clear cell renal cell carcinoma
}

\author{
Yejinpeng Wang ${ }^{1}$, Liang Chen ${ }^{1}$, Lingao Ju 2,3,4, Kaiyu Qian²,3,4, Xinghuan Wang ${ }^{1,5}$, Yu Xiao 1,2,3,4,6 \\ and Gang Wang $2,3,4^{*}$ (D)
}

\begin{abstract}
Background: Recently, increasing study have found that DNA methylation plays an important role in tumor, including clear cell renal cell carcinoma (ccRCC).

Methods: We used the DNA methylation dataset of The Cancer Genome Atlas (TCGA) database to construct a 31-CpG-based signature which could accurately predict the overall survival of ccRCC. Meanwhile, we constructed a nomogram to predict the prognosis of patients with cCRCC.

Result: Through LASSO Cox regression analysis, we obtained the 31-CpG-based epigenetic signature which were significantly related to the prognosis of ccRCC. According to the epigenetic signature, patients were divided into two groups with high and low risk, and the predictive value of the epigenetic signature was verified by other two sets. In the training set, hazard ratio $(\mathrm{HR})=13.0,95 \%$ confidence interval $(\mathrm{Cl}) 8.0-21.2, P<0.0001$; testing set: $\mathrm{HR}=4.1, \mathrm{Cl}$ 2.2-7.7, $P<0.0001$; entire set: $H R=7.2, C l$ 4.9-10.6, $P<0.0001$, Moreover, combined with clinical indicators, the prediction of 5 -year survival of cCRCC reached an AUC of 0.871 .

Conclusions: Our study constructed a 31-CpG-based epigenetic signature that could accurately predicted overall survival of $c C R C C$ and staging progression of $C C R C C$. At the same time, we constructed a nomogram, which may facilitate the prediction of prognosis for patients with ccRCC.
\end{abstract}

Keywords: Epigenetic signature, Clear cell renal cell carcinoma, Overall survival, Methylation, Nomogram

\section{Background}

Renal cell carcinoma (RCC) is a cancer that originates in the renal epithelial cells and accounts for more than $90 \%$ of renal cancer, of which clear cell RCC (ccRCC) is most common subtype and causes the most deaths [1]. According to statistics, ccRCC caused 14,400 deaths in 2017 [2]. The TNM staging system is still the most commonly used clinical tool to stratify ccRCC patients, however, it is not enough to reflect the biological heterogeneity of tumor and accurately predict the prognosis of ccRCC patients

*Correspondence: gangwang.uro@whu.edu.cn

${ }^{2}$ Department of Biological Repositories, Zhongnan Hospital of Wuhan University, Wuhan, China

Full list of author information is available at the end of the article
[3], and a better marker is urgently needed to help us to accurately predict.

The aberrant methylation status of $\mathrm{CpG}$ islands located in the promoter region of tumor genes is becoming increasingly important in the search for new potential biomarkers for cancer [4-6], because these aberrant are relatively stable and potentially reversible [7]. Combining multiple markers, rather than using only one marker to construct a prognostic model, will make the results more stable and reliable, and improve its predictive value [8]. Many studies have used multiple targets to construct the prognostic model of ccRCC $[9,10]$. Here, we used the methylation data of the ccRCC of TCGA database to construct a 31-CpG-based signature.

In this study, we used the methylation data of the ccRCC of TCGA database to construct a 31-CpG-based

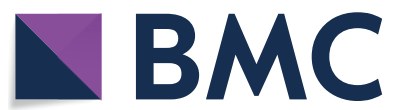

(c) The Author(s) 2020. This article is licensed under a Creative Commons Attribution 4.0 International License, which permits use, sharing, adaptation, distribution and reproduction in any medium or format, as long as you give appropriate credit to the original author(s) and the source, provide a link to the Creative Commons licence, and indicate if changes were made. The images or other third party material in this article are included in the article's Creative Commons licence, unless indicated otherwise in a credit line to the material. If material is not included in the article's Creative Commons licence and your intended use is not permitted by statutory regulation or exceeds the permitted use, you will need to obtain permission directly from the copyright holder. To view a copy of this licence, visit http://creativeco mmons.org/licenses/by/4.0/. The Creative Commons Public Domain Dedication waiver (http://creativecommons.org/publicdomain/ zero/1.0/) applies to the data made available in this article, unless otherwise stated in a credit line to the data. 
epigenetic signature, which can accurately predict the overall survival rate of ccRCC through the most recently used The Least Absolute Shrinkage And Selection Operator method (LASSO) algorithm. We then verified this epigenetic marker using two validation sets (testing set and entire set). Furthermore, we constructed a nomogram to facilitate clinicians to accurately predict overall survival in ccRCC patients. Our results identified a new 31-CpG-based epigenetic marker that may be a new target for predicting overall survival of ccRCC.

\section{Methods}

\section{Study population and data collection}

A total of 319 cases of ccRCC were randomly assigned to two groups, which had a 2:1 ratio. The former was defined as the training set $(n=213)$ and the latter as testing set $(n=106)$. The corresponding clinical follow-up information of all cases included the survival time, survival outcome, tumor staging, grading and other information of the cases. The level three of RNA-seq (Illumina RNASeqV2) and the Infinium HumanMethylation 450 BeadChip array (Illumina) data were downloaded from The Cancer Genome Atlas (TCGA) database (http:// cancergenome.nih.gov/), The details were listed in Table 1, and the flow chart of our entire experiment was shown in Fig. 1.

\section{Differentially methylated positions screening}

We first filtered out the probes that have too many zeros, then used the R package "ChAMP" [11] to quality control, remove the batch effects, BMIQ normalization [12] and Single Nucleotide Polymorphism (SNP) filtering (if it located in the CpG dinucleotide) [13]. After that, we used "champ.DMP" function to perform differentially methylated positions (DMP) analysis, we chosen the false discovery rate $(\mathrm{FDR})<0.05$ and $|\Delta \beta| \geq 0.2$ as the threshold for screening DMPs.

\section{Establishment and validation of epigenetic signature}

LASSO cox regression analysis was conducted for the DMPs obtained in the previous step, based on R packet "glmnet". The degree of LASSO regression complexity adjustment was controlled by the parameter $\lambda$, and the larger $\lambda$ was, the greater the penalty was, so as to obtain a model with fewer variables [14]. We construct the model according to the protocol of official website (https:// cran.r-project.org/web/packages/glmnet/glmnet.pdf). Frist, the function "glmnet" returned a sequence of models for us to choose from. Second, we used the function "cv.glmnet" to perform Cross-validation, we followed the protocol, did 100 times cross-validations, and finally got an $\lambda$ average. Based on this $\lambda$ value, we constructed an epigenetic signature based on $31 \mathrm{CpGs}$ from training
Table 1 Clinical characteristics of ccRCC patients

\begin{tabular}{|c|c|c|c|c|c|}
\hline \multirow[t]{3}{*}{ Variable } & \multirow{3}{*}{$\begin{array}{l}\text { Training set } \\
n=213 \\
\text { No. (\%) }\end{array}$} & \multirow{3}{*}{$\begin{array}{l}\text { Testing set } \\
n=106 \\
\text { No. (\%) }\end{array}$} & \multirow{3}{*}{$\begin{array}{l}\text { Entire set } \\
n=319 \\
\text { No. (\%) }\end{array}$} & \multicolumn{2}{|c|}{$\begin{array}{l}\text { Chi-square } \\
\text { Test }\end{array}$} \\
\hline & & & & \multirow[t]{2}{*}{$\mathrm{x}^{2}$} & \multirow[t]{2}{*}{$P$} \\
\hline & & & & & \\
\hline Censor & $65(30.5)$ & $40(37.7)$ & $105(32.9)$ & & \\
\hline \multicolumn{6}{|l|}{ Age (years) } \\
\hline$\geq 65$ & 75 (35.2) & $36(34.0)$ & $111(34.8)$ & \multirow[t]{2}{*}{0.049} & \multirow[t]{2}{*}{0.825} \\
\hline$<65$ & $138(64.8)$ & $70(66.0)$ & $208(65.2)$ & & \\
\hline \multicolumn{6}{|l|}{ Gender } \\
\hline Female & $80(37.6)$ & $34(32.1)$ & $114(35.7)$ & \multirow[t]{2}{*}{0.927} & \multirow[t]{2}{*}{0.336} \\
\hline Male & $133(62.4)$ & $72(67.9)$ & $205(64.3)$ & & \\
\hline \multicolumn{6}{|c|}{ Pathological stage } \\
\hline Stage I & $106(49.8)$ & $49(46.2)$ & $155(48.6)$ & \multirow[t]{5}{*}{1.766} & \multirow[t]{5}{*}{0.779} \\
\hline Stage II & $22(10.3)$ & $9(8.5)$ & $31(9.7)$ & & \\
\hline Stage III & $49(23.0)$ & $24(22.6)$ & $73(22.9)$ & & \\
\hline Stage IV & $35(16.4)$ & $23(21.7)$ & $58(18.2)$ & & \\
\hline NA & $1(0.5)$ & $1(0.9)$ & $2(0.6)$ & & \\
\hline \multicolumn{6}{|c|}{ Histologic grade } \\
\hline Grade I & $6(2.8)$ & $3(2.8)$ & $9(2.8)$ & \multirow[t]{5}{*}{4.684} & \multirow[t]{5}{*}{0.321} \\
\hline Grade II & $93(43.7)$ & $40(30.7)$ & $133(41.7)$ & & \\
\hline Grade III & $75(35.2)$ & $48(45.3)$ & $123(38.6)$ & & \\
\hline Grade IV & $35(16.4)$ & $15(14.2)$ & $50(15.7)$ & & \\
\hline NA & $4(1.9)$ & $0(0.0)$ & $4(1.3)$ & & \\
\hline \multicolumn{6}{|c|}{ Lymph node metastasis } \\
\hline Positive & $38(17.8)$ & $23(21.7)$ & $61(19.1)$ & \multirow[t]{2}{*}{0.681} & \multirow[t]{2}{*}{0.409} \\
\hline Negative & $175(82.2)$ & $83(78.3)$ & $258(80.9)$ & & \\
\hline
\end{tabular}

Here Chi-square and $p$-value were the values obtained by comparison between the training set and the testing set

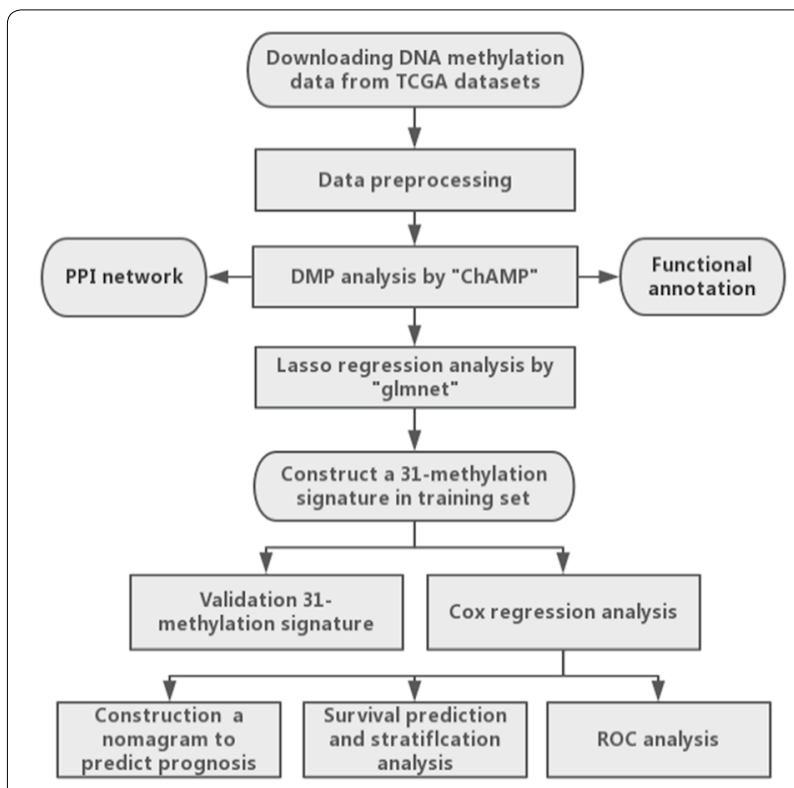

Fig. 1 Flow diagram of the study 
set. The accuracy of the epigenetic signature was then verified in the testing set and the entire set by using the time-dependent ROC curve and Kaplan-Meier survival curve analysis. Time-dependent ROC curve analysis was performed by R package "survivalROC" [15] while the Kaplan-Meier survival curve analysis was performed by R package "survival".

\section{Cox regression analysis of the epigenetic signature}

To investigate whether the epigenetic signature we constructed could be independent of other factors affecting the prognosis of ccRCC, we conducted univariate and multivariate analyses by R package "survival". First of all, the epigenetic signature score, age, gender, neoadjuvant treatment, lymph node count, histologic stage and pathologic grade were performed the univariate analysis. Then, we put the univariate analysis has significant difference factor to carry on the multivariate analysis. Finally, we used the forest map to visualize our results by $\mathrm{R}$ package "forestplot".

\section{The construction of the nomogram}

Based on the results of the previous step, we used the factors with significant differences (epigenetic signature score and histologic stage) in multivariate regression analysis to construct a nomogram by using the $\mathrm{R}$ package "rms". In order to verify the accuracy of the nomogram, we used the calibration curve to evaluate the nomogram. In the calibration curve, if the observed value and the actual value are more coincident, it indicates that the prediction accuracy of the nomogram is higher.

\section{Functional annotation of the epigenetic signature}

We calculated the top 500 genes with the highest correlation with epigenetic signature score, and then enriched the functions of these 500 genes, thus indirectly predicting the functional annotation of epigenetic marker score. The 500 genes were uploaded to the DAVID website (https://david.ncifcrf.gov/) for GO analysis and KEGG pathway analysis. The following, the STRING database (https://string-db.org/) was used to performed protein-protein interaction (PPI) network [16] analysis. We've picked the top 50 genes from the "cytoHubba" app at Cytoscape and used the Cytoscape to visualize the results. According to the scores of the epigenetic signature, patients were divided into two groups with high and low risk, and then the corresponding RNA-seq data of these two groups were analyzed by single-samples gene-set enrichment analysis. The R package "GSVA" [17] were used to perform ssGSEA for the 31-CpGs-based epigenetic signature. The C2 (c2.cp.kegg.v6.1.symbols. gmt) set was downloaded from the Molecular Signatures
Database (http://software.broadinstitute.org/gsea/msigd $\mathrm{b} /$ index.jsp). And it was chosen as the signature to perform ssGSEA. The R package "limma" was used to identify the differentially expressed gene sets. The threshold for screening differentially expressed gene sets were the p-value $<0.05$.

\section{Results}

\section{Screening of DMPs}

The R package "ChAMP" was used to screen DMPs between ccRCC and normal ccRCC samples in ccRCC dataset of the TCGA database, a total of 3858 DMPs were identified (1641 up-regulated and 576 down-regulated), under the threshold of FDR $<0.05$ and $|\Delta \beta| \geq 0.2$. All DMPs were listed in Additional file 1: Table S1.

\section{Establishment and validation of the epigenetic signature}

We used the R package of "glmnet" to construct LASSO cox regression model. After 100 cross - validation, we got an optimal $\lambda$ value $(\lambda=0.054)$, and finally we constructed an epigenetic signature based on $31 \mathrm{CpGs}$. The formula of epigenetic signature score were obtained by calculating the LASSO regression coefficient: epigenetic signature score $=\sum_{k=1}^{n} \beta_{k i} X_{k i}$, here, $n$ is the number of the CpG site; $\beta$ is the LASSO regression coefficient of the CpG site; $X$ is the methylation value of $\mathrm{CpG} k$ and patient $i ; k$ is the CpG site. Details of the $31 \mathrm{CpGs}$ were listed in Table 2, the heatmap of the 31 CpGs were shown in Fig. $2 \mathrm{a}$, and the results of ten-time cross-validation were shown in Fig. $2 b$.

We divided the patients in the training set into high and low risk groups based on the median epigenetic signature scores (here, the median epigenetic signature score was 2.96, Fig. 3a, left panel). Then, time-dependent ROC curve analysis revealed the epigenetic signature we constructed were able to predict the overall survival rate of patients with ccRCC with great accuracy. The AUC of time-dependent ROC for 1-year overall survival was 0.875 , 3-year $0.876,5$-year 0.851 and 7 -year 0.855 (Fig. 3b middle panel). Finally, Kaplan-Meier survival curve analysis showed that the high-risk group had a significantly worse prognosis than the low-risk group (HR $=13.0,95 \%$ CI $8.0-21.2, P<0.0001$, Fig. 3a right panel). In order to avoid over-fitting effect, testing set $(\mathrm{HR}=4.1$, CI 2.2-7.7, $P<0.0001$, Fig. 3b) and entire set $(\mathrm{HR}=7.2$, CI 4.9-10.6, $P<0.0001$, Fig. 3c) were used for verification our results.

\section{Univariate and multivariate regression analysis of the epigenetic signature}

We first performed a univariate analysis of the epigenetic signature scores, age, gender, neoadjuvant treatment, 
Table 2 31-CpG-sites details in the epigenetic signature

\begin{tabular}{|c|c|c|c|c|c|c|c|}
\hline CpG ID & Gene symbol & CHR & Strand & Type & Feature & Delta $\beta$ & Lasso coef \\
\hline cg00936626 & PIGZ & 3 & F & $\|$ & 5'UTR & -0.20 & 0.96 \\
\hline cg01569664 & IRS2 & 13 & $\mathrm{R}$ & I & Body & -0.20 & 0.2 \\
\hline cg03429569 & & 2 & $\mathrm{R}$ & $\|$ & IGR & -0.22 & -0.31 \\
\hline cg03615683 & & 12 & $\mathrm{R}$ & $\|$ & IGR & -0.21 & 0.62 \\
\hline cg04025970 & MFHAS1 & 8 & $\mathrm{R}$ & $\|$ & Body & -0.23 & 0.12 \\
\hline cg04074945 & PHF21A & 11 & $\mathrm{~F}$ & $\|$ & Body & -0.26 & -0.06 \\
\hline cg075222913 & HOXA3 & 7 & $\mathrm{~F}$ & । & 5'UTR & -0.27 & -0.25 \\
\hline cg07915516 & AXIN1 & 16 & $\mathrm{R}$ & $\|$ & Body & 0.22 & 0.67 \\
\hline cg08699206 & & 4 & $\mathrm{R}$ & I & IGR & -0.34 & 0.93 \\
\hline cg08949329 & COL4A2 & 13 & $\mathrm{R}$ & $\|$ & Body & -0.22 & 0.6 \\
\hline cg09507567 & & 10 & $\mathrm{R}$ & $\|$ & IGR & 0.24 & 0.06 \\
\hline cg09744051 & A4GALT & 22 & $F$ & $\|$ & $5^{\prime} U T R$ & 0.25 & 0.59 \\
\hline cg10057940 & & 10 & $F$ & $\|$ & IGR & -0.24 & 0.57 \\
\hline $\operatorname{cg} 10621809$ & & 12 & $\mathrm{R}$ & $\|$ & IGR & -0.24 & 0.47 \\
\hline cg12304520 & PCDHGA4 & 5 & $\mathrm{R}$ & $\|$ & Body & -0.22 & 0.7 \\
\hline cg12864389 & PLEC1 & 8 & $\mathrm{~F}$ & $\|$ & Body & 0.37 & -1.15 \\
\hline cg14476745 & LHX6 & 9 & $\mathrm{R}$ & I & Body & -0.31 & 0.1 \\
\hline cg15022051 & & 6 & $\mathrm{R}$ & I & IGR & -0.25 & 0.48 \\
\hline cg15518113 & CD247 & 1 & $\mathrm{R}$ & $\|$ & $3^{\prime}$ UTR & -0.22 & 1.09 \\
\hline cg16059943 & PRKCZ & 1 & $\mathrm{~F}$ & $\|$ & Body & -0.23 & -1.38 \\
\hline cg16342949 & MACROD1 & 11 & F & I & Body & -0.24 & 0.38 \\
\hline cg16723800 & TAF7L & $x$ & F & 1 & Body & -0.31 & 0.04 \\
\hline cg17482089 & PCDHA6 & 5 & R & I & Body & -0.24 & 0.73 \\
\hline cg18954144 & & 2 & $\mathrm{R}$ & I & IGR & -0.30 & 0.43 \\
\hline cg19476788 & NFATC1 & 18 & $\mathrm{R}$ & I & Body & -0.26 & 0.08 \\
\hline cg19528338 & BMP2 & 20 & $\mathrm{R}$ & $\|$ & 5'UTR & 0.21 & 0.6 \\
\hline cg21033440 & SIPA1 & 11 & F & I & Body & -0.26 & 0.65 \\
\hline cg21157873 & CGN & 1 & R & $\|$ & $5^{\prime}$ UTR & -0.26 & 0.03 \\
\hline cg24997744 & BAHCC 1 & 17 & $\mathrm{R}$ & I & 3'UTR & -0.22 & -1.27 \\
\hline cg25541653 & MPPED2 & 11 & R & $\|$ & Body & -0.25 & -1.29 \\
\hline cg25755851 & & 1 & $\mathrm{~F}$ & $\|$ & IGR & 0.41 & -0.13 \\
\hline
\end{tabular}

lymph node examination, histologic grade and pathologic stage in the entire set, and the results revealed that only the epigenetic signature score $(\mathrm{HR}=3.87,95 \% \mathrm{CI}$ 3.02-4.97, $P<0.0001)$, age ( $\mathrm{HR}=1.5,95 \%$ CI $1.02-2.21$, $P=0.0378)$, histologic grade $(\mathrm{HR}=3.35,95 \% \mathrm{CI} 2.07-$ 5.41, $P<0.0001)$ and pathologic stage $(\mathrm{HR}=4.49,95 \% \mathrm{CI}$ 2.92-6.89, $P<0.0001$ ) have significant prognostic value (Fig. 4a). Next, we conducted multivariate analysis of the factors with significant differences above, and found that the epigenetic signature score $(\mathrm{HR}=3.09$, 95\% CI 2.354.08, $P<0.0001)$ and pathologic stage $(\mathrm{HR}=2.42,95 \% \mathrm{CI}$ $1.52-3.86, P=0.0002)$ could accurately predict the overall survival rate of ccRCC independently of other factors (Fig. 4b, Table 3).

\section{Nomogram creating and calibrating}

In order to more conveniently predict the overall survival rate of ccRCC patients for clinicians, we constructed a nomogram based on the $\mathrm{R}$ package "rms". This nomogram could accurately predict 3- or 5-year overall survival in patients with ccRCC (Fig. 5a). Moreover, we used the calibration curve to calibrate the nomogram and found that the prediction value of nomogram was significantly correlated with the actual value (Fig. 5b, c).

\section{Subgroup analysis of the epigenetic signature}

To explore the predictive value of epigenetic signature in different subgroups, we performed subgroup analyzed 


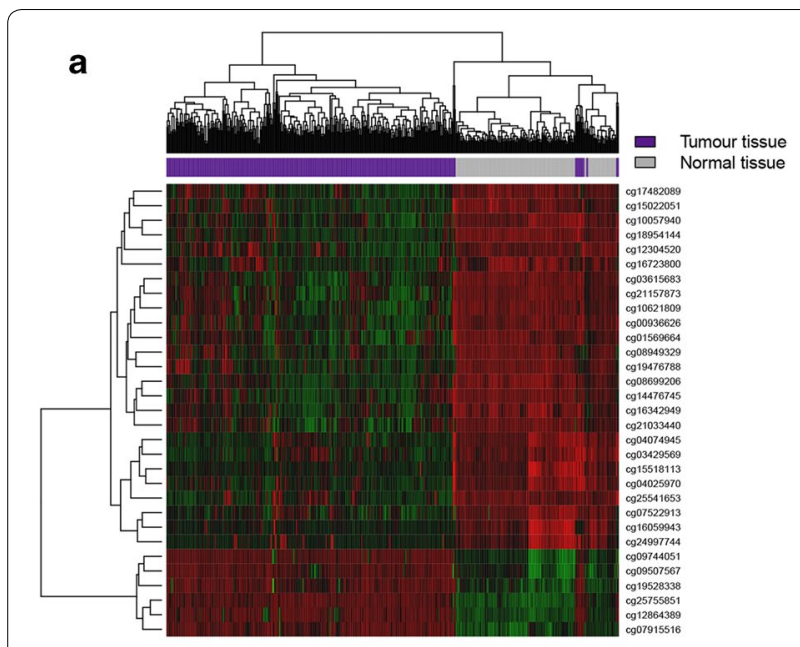

$\begin{array}{llllllllllll}\text { b } & 114 & 121 & 111 & 109 & 97 & 78 & 49 & 29 & 16 & 6 & 2\end{array}$

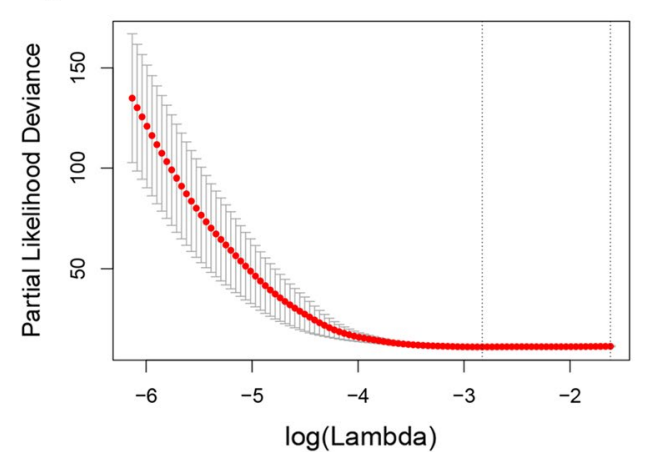

Fig. 2 Establishment of epigenetic signature based on 31-CpG-sites. a Heatmap of the 31-CpG-sites that were used to construct the epigenetic signature. $\mathbf{b}$ Ten-time cross-validation for parameter chosen. Here, $\lambda=0.054$ was chosen by ten-time cross-validation

patients from the entire set according to different characteristics: pathologic stage (III/IV vs I/II, Fig. 6a, b), histologic grade (III/IV vs I/II, Fig. 6c, d), lymph node examination (Positive vs negative, Fig. 6e, f) and age ( $>=65$ vs $<65$, Fig. $6 \mathrm{~g}, \mathrm{~h}$ ). The detailed information of subgroup analysis for the epigenetic signature were listed in Table 4.

\section{Functional annotation of the epigenetic signature}

Pearson correlation coefficients of all genes and the epigenetic signature scores were calculated. The 500 genes with the highest correlation were selected for subsequent analysis, and the threshold value was set as p-value less than 0.05 . Functional enrichment of the 500 genes showed that this epigenetic signature was mainly enriched in the cell adhesion, notch signaling pathway, axon guidance, thyroid hormone signaling pathway and so on (Additional file 2: Figure S1). The PPI network diagram we built was shown in Additional file 3: Figure S2.

After the enrichment scores of each sample were calculated, the differences of the high and low groups were analyzed according to the epigenetic signature scores. A total of 26 differentially expressed gene sets (10 up-regulated and 16 down-regulated) were screened under the threshold $\mathrm{FDR}<0.05$ and $|\operatorname{LogFC}|>0.015$. The result of ssGSEA revealed that patients in high risk group mainly enriched in p53, nod like receptor, cytosolic DNA sensing and other signaling pathways. The patients in low risk group were mainly enriched in the fatty acid metabolism pathway, PPAR signaling pathway, renin angiotensin system and so on (Fig. 7c, Additional file 1: Table S2).

\section{Verification of CpG island methylation levels in ccRCC tissues and renal carcinoma cell lines}

We collected 4 samples diagnosed as ccRCC and 2 paracancer tissue from the Zhongnan Hospital of Wuhan University, plus human renal tubular epithelial cell line (HK2) and 2 kidney cancer cell lines (ACHN and 769-P), and sequenced them with targeted methylation (MethylTarget, Illumina Hiseq, Illumina, CA, USA). The results showed that $\mathrm{CpG}$ island involved in the construction of prognostic model had different expression patterns between carcinoma and paracancer (Fig. 7d).

\section{Discussion}

Patients with ccRCC exhibit a highly invasive clinical process, poor prognosis, and high recurrence rate [1]. The TNM staging system is still widely used to predict the prognosis of ccRCC, however, it relies mainly on anatomical information and has no biological characteristics [9]. Therefore, we need a new target to accurately predict the prognosis of ccRCC.

Overfitting often occurs in selecting prognostic models using higher-dimensional data [18], here, we chosen the LASSO algorithm, which could eliminate this disadvantage [19], as the method to select CpG for constructing epigenetic signature. The algorithm of LASSO has been widely used to construct predictive survival models, for instance, colon cancer [20], gastric cancer [21], T-cell lymphoblastic lymphoma [22] and ccRCC $[9,10$, 23].

In recent years, epigenetics has been studied more and more in ccRCC, DNA methylation is one of the most studied patterns in epigenetic regulation [24-26]. And there have also been a number of studies using 

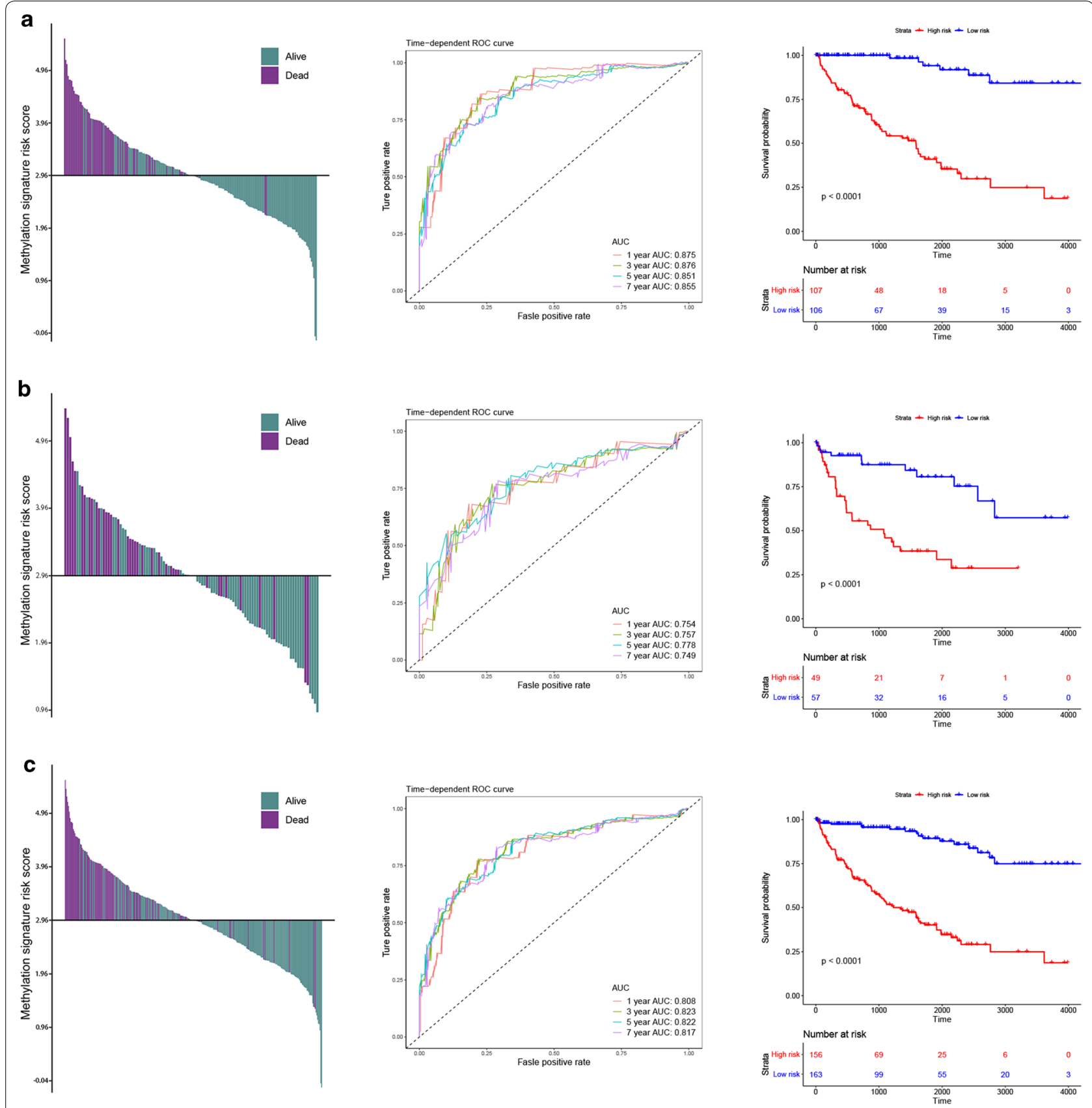

Fig. 3 Risk scores by the epigenetic signature, the time-dependent ROC curves and Kaplan-Meier survival curves in the training set, testing set and entire set. a Training set. b Testing set. $\mathbf{c}$ Entire set. The AUCs at 1, 3, 5, and 7 years were used to assess the prognostic accuracy, and the log-rank test was used to calculated the $p$-values

methylation data to construct prognostic signatures [5, $6,27]$. Here, we constructed and validated a 31-CpGbased epigenetic signature that could accurately predicted overall survival of ccRCC. Using the epigenetic signature we constructed, we divided patients into high and low risk groups, and high-risk patients had significantly lower overall survival rates in each set. Although previous studies have constructed several molecular 


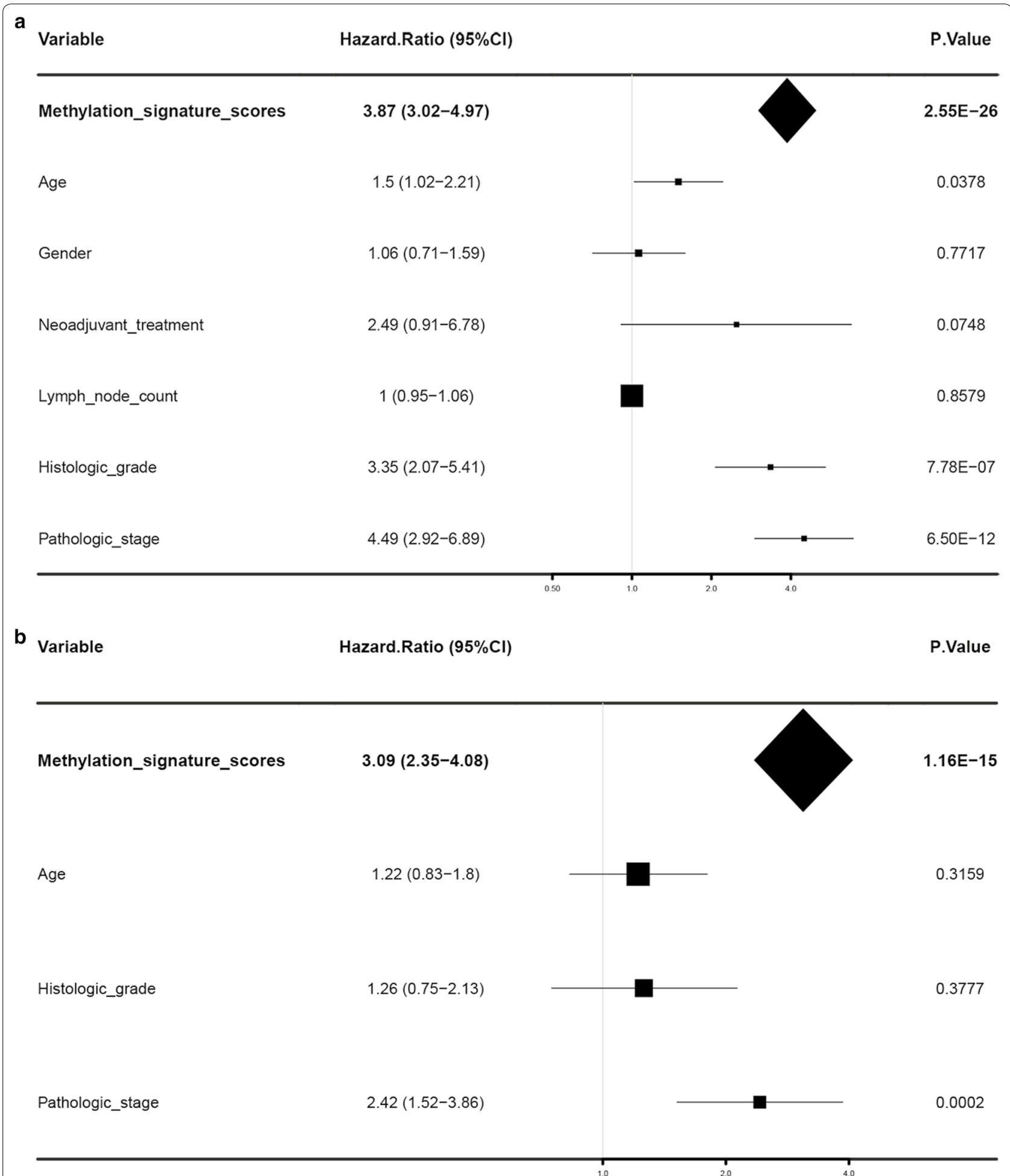

Fig. 4 Forest plot of univariable and multivariable analysis. Univariable analysis (a) and multivariable analysis (b) of the methylation signature scores, age, gender, histologic grade, and so on 
Table 3 Univariate analysis and multivariate analysis of the epigenetic signature

\begin{tabular}{|c|c|c|c|c|c|c|}
\hline \multirow[t]{2}{*}{ Variable } & \multicolumn{3}{|c|}{ Univariate analysis } & \multicolumn{3}{|c|}{ Multivariate analysis } \\
\hline & HR & $95 \% \mathrm{Cl}$ & $P$ & HR & $95 \% \mathrm{Cl}$ & $P$ \\
\hline MS scores & 3.87 & $(3.02-4.97)$ & $<0.001$ & 3.09 & $(2.35-4.08)$ & $<0.001$ \\
\hline Age & 1.5 & $(1.02-2.21)$ & 0.038 & 1.22 & $(0.83-1.8)$ & 0.316 \\
\hline Gender & 1.06 & $(0.71-1.59)$ & 0.772 & & & \\
\hline Neoadjuvant treatment & 2.49 & $(0.91-6.78)$ & 0.075 & & & \\
\hline Lymph node count & 1 & $(0.95-1.06)$ & 0.858 & & & \\
\hline Histologic grade & 3.35 & $(2.07-5.41)$ & $<0.001$ & 1.26 & $(0.75-2.13)$ & 0.378 \\
\hline Pathologic stage & 4.49 & $(2.92-6.89)$ & $<0.001$ & 2.42 & $(1.52-3.86)$ & $<0.001$ \\
\hline
\end{tabular}

a

Points

Risk scores

Stage

Total Points

Linear Predictor

1-year OS

3-year OS

5-year OS

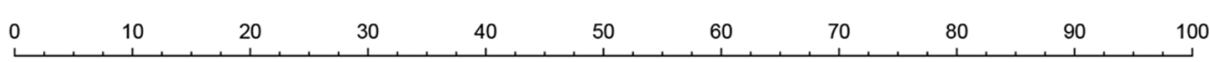

\begin{tabular}{llllllllllllll}
\hline 6 & 5.5 & 5 & 4.5 & 4 & 3.5 & 3 & 2.5 & 2 & 1.5 & 1 & 0.5 & 0 & -0.5 \\
& & $\mathrm{I} / \mathrm{II}$ & & & & & & & & & &
\end{tabular}

III/IV
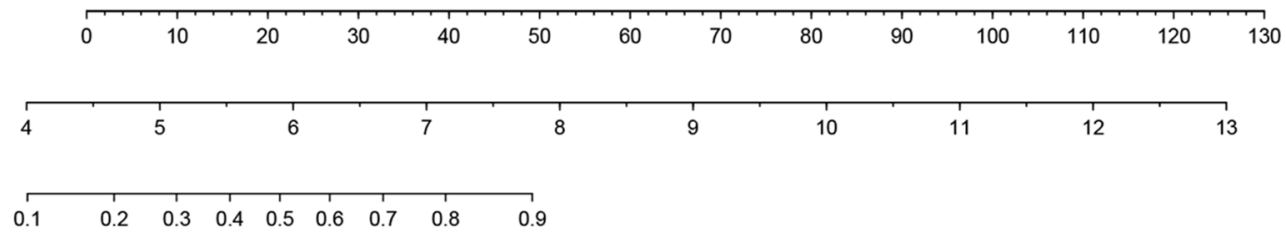

b
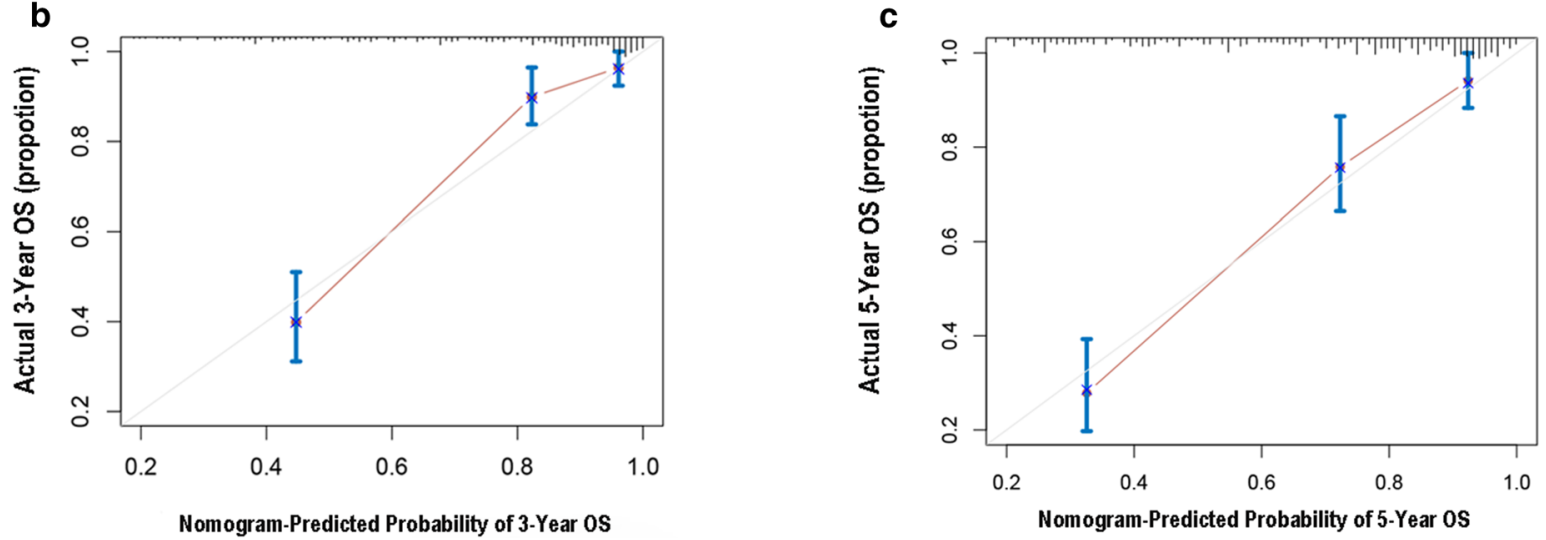

Fig. 5 The nomogram to predict the overall survival of ccRCC. a The nomograms for predicting proportion of ccRCC patients with 1-, 3- or 5-year overall survival. Plots depict the calibration of the nomogram between predicted and observed 3- (b) or 5- (c) year outcomes

(See figure on next page.)

Fig. 6 Kaplan-Meier survival analysis for entire set according to the 31-CpG-sites based epigenetic signature stratified by clinicopathological risk factors. a, b Pathologic stage. c, d Histologic grade. e, $\mathbf{f}$ Results of lymph nodes examined. $\mathbf{g}, \mathbf{h}$ Age 


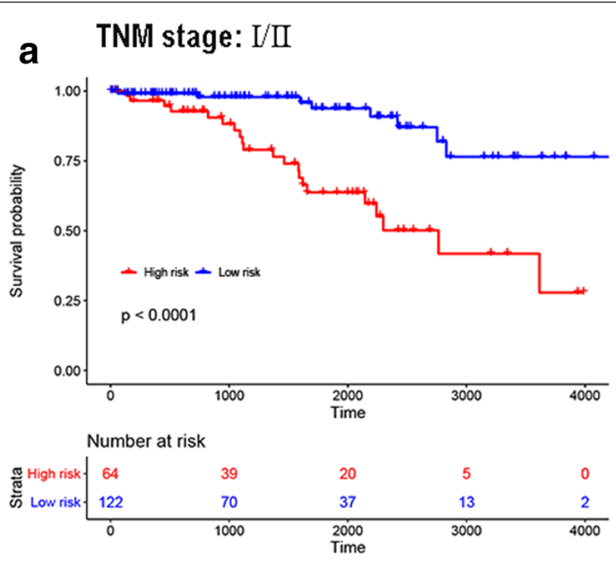

b TNM stage: $\mathrm{II} / \mathrm{IV}$

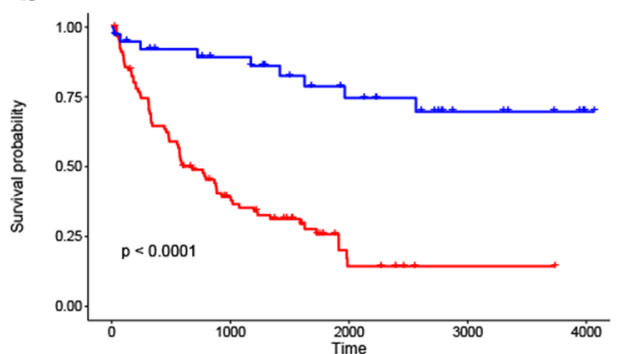

C Tumour grade: low

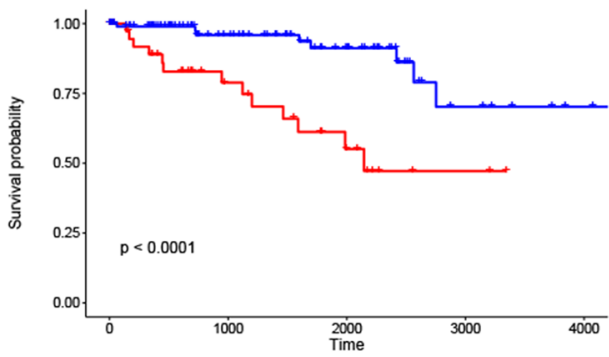

d Tumour grade: high
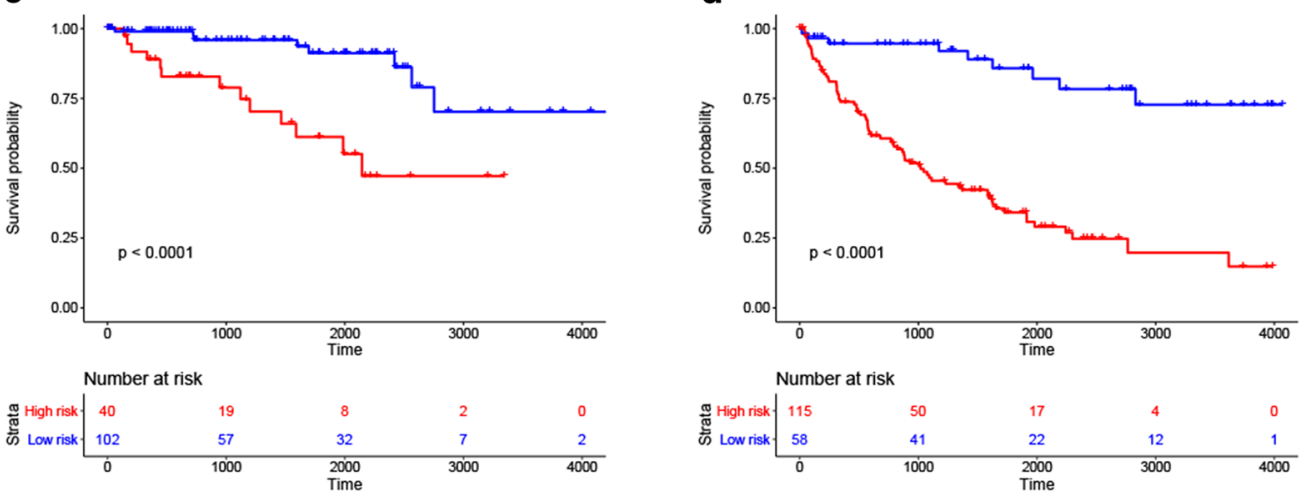

\section{e Nodes examined: positive}
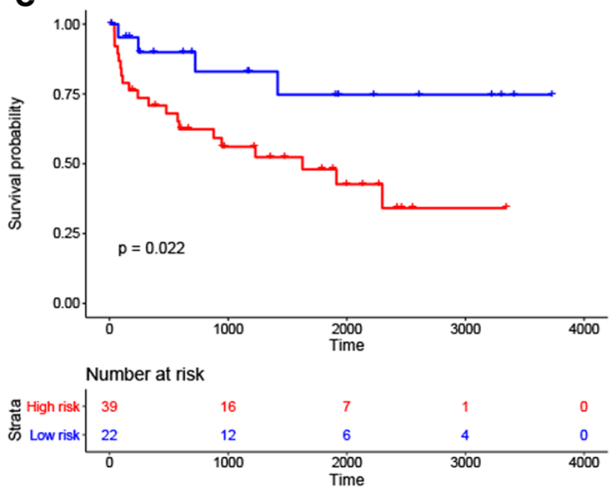

Age: $\geq 65$

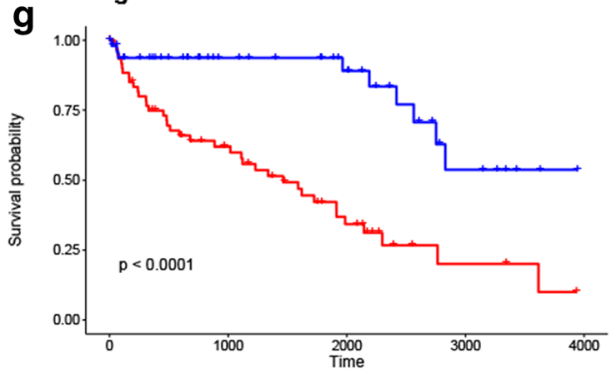

Number at risk

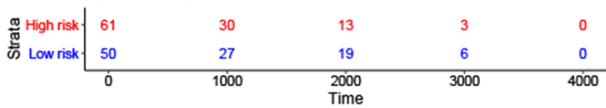

\section{f Nodes examined: negative}
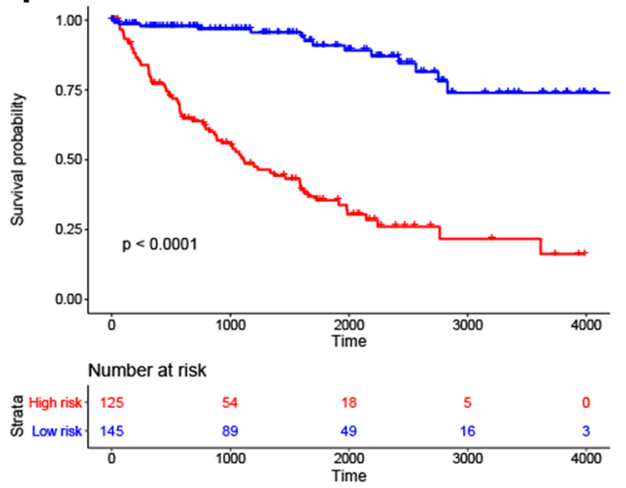

Age: $<65$

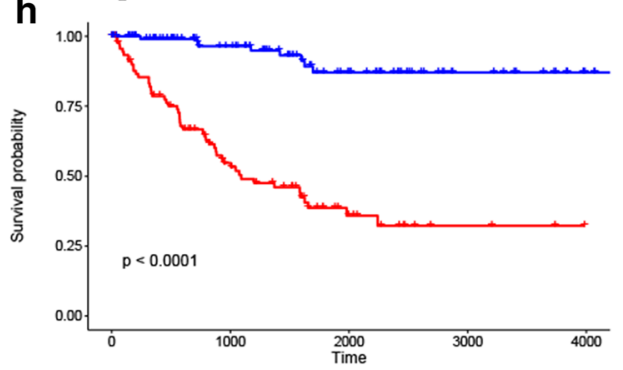

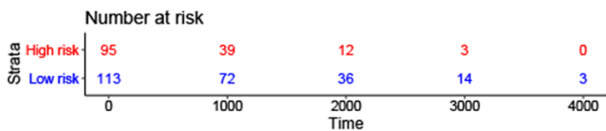


Table 4 Kaplan-Meier survival analysis for the epigenetic signature in these three sets

\begin{tabular}{|c|c|c|c|c|c|c|}
\hline \multirow[t]{2}{*}{ Variable } & \multicolumn{2}{|c|}{ Training set $(n=213)$} & \multicolumn{2}{|c|}{ Testing set $(n=106)$} & \multicolumn{2}{|c|}{ Entire set $(n=319)$} \\
\hline & HR $(95 \% \mathrm{Cl})$ & $P$ value & HR $(95 \% \mathrm{Cl})$ & $P$ value & HR $(95 \% \mathrm{Cl})$ & $P$ value \\
\hline All & $13.0(8.0-21.2)$ & $<0.0001$ & $4.1(2.2-7.7)$ & $<0.0001$ & $7.2(4.9-10.6)$ & $<0.0001$ \\
\hline \multicolumn{7}{|l|}{ Age (years) } \\
\hline$\geq 65$ & $7.6(3.7-15.8)$ & $<0.0001$ & $2.9(1.2-7.4)$ & 0.0178 & $4.5(2.5-8.0)$ & $<0.0001$ \\
\hline$<65$ & $16.8(8.6-32.8)$ & $<0.0001$ & $5.4(2.3-12.7)$ & 0.0002 & $9.6(5.7-16.3)$ & $<0.0001$ \\
\hline \multicolumn{7}{|l|}{ Gender } \\
\hline Female & $15.7(6.8-36.4)$ & $<0.0001$ & $4.5(1.2-16.6)$ & 0.0071 & $9.3(4.6-18.8)$ & $<0.0001$ \\
\hline Male & $12.1(6.5-22.5)$ & $<0.0001$ & $4.0(1.9-8.2)$ & 0.0005 & $6.4(4.0-11.2)$ & $<0.0001$ \\
\hline \multicolumn{7}{|c|}{ Pathological stage } \\
\hline Stage $|/| \mid$ & $10.4(4.3-25.3)$ & $<0.0001$ & - & 0.4414 & $5.2(2.4-11.4)$ & $<0.0001$ \\
\hline Stage III/IV & $10.7(5.9-19.3)$ & $<0.0001$ & $2.5(1.2-5.2)$ & 0.0258 & $5.3(3.3-8.3)$ & $<0.0001$ \\
\hline \multicolumn{7}{|c|}{ Histologic grade } \\
\hline Grade I/II & $10.6(3.0-38.1)$ & $<0.0001$ & - & 0.5311 & $5.1(1.9-13.8)$ & $<0.0001$ \\
\hline Grade III/IV & $10.1(5.8-17.8)$ & $<0.0001$ & $3.9(2.0-7.9)$ & 0.0007 & $6.1(3.9-6.4)$ & $<0.0001$ \\
\hline \multicolumn{7}{|c|}{ Lymph node metastasis } \\
\hline Positive & - & 0.0048 & - & 0.5651 & $3.3(1.4-7.4)$ & 0.0222 \\
\hline Negative & $12.2(7.1-21.0)$ & $<0.0001$ & $5.5(2.5-12.3)$ & $<0.0001$ & $8.6(5.5-13.5)$ & $<0.0001$ \\
\hline
\end{tabular}

biomarkers to predict the survival of ccRCC, few of the signature they have constructed are as accurate as our epigenetic signature. The AUC of time-dependent ROC was all greater than 0.75 in each set. After the epigenetic signature combined with staging and grading, the AUC predicted 5-year overall survival was reached 0.871 . At the same time, we validated our epigenetic signature in different subgroups (pathologic stage, histologic grade, lymph node examination, and age) and found that the epigenetic signature showed excellent prognostic value.

We use ssGSEA and other methods to infer the functional annotation of epigenetic signature, and we found that the high risk group patients were mainly enriched in p53 and nod like receptor signaling pathway, which have been reported in previous studies to be associated with the pathogenesis of ccRCC [28-30]. The group of low risk were enriched in fatty acid metabolism and which has also been reported to be associated with ccRCC [31-33]. We speculate that these pathways may be responsible for the very different overall survival rates between the two groups based on the epigenetic signature. So what we're going to do is to look at how do these pathways affect the prognosis of ccRCC.
We performed methylation sequencing on ccRCC tissue samples and kidney cancer cell lines, and the results showed that CpG islands involved in the construction of the prognosis model had significantly different expression patterns. However, our study has some limitations, we lack more data to verify it, but we have built a new biological sample database called Biological Repositories Zhongnan Hospital of Wuhan University (http://bioba nk.znhospital.cn), which will provide a lot of data to verify our epigenetic markers in the future.

In conclusion, we provide an effective epigenetic signature that accurately predicts overall survival of ccRCC independent of other risk factors (age, gender, neoadjuvant treatment, lymph node examination, histologic grade and pathologic stage). At the same time, we also constructed a nomogram for the convenience of clinicians to predict the prognosis of ccRCC.

\section{Conclusion}

We developed a 31-CpG-based signature using bioinformatics methods (LASSO cox regression analysis). We found that this epigenetic signature could accurately predict the overall survival rate of ccRCC, contributing to 
a

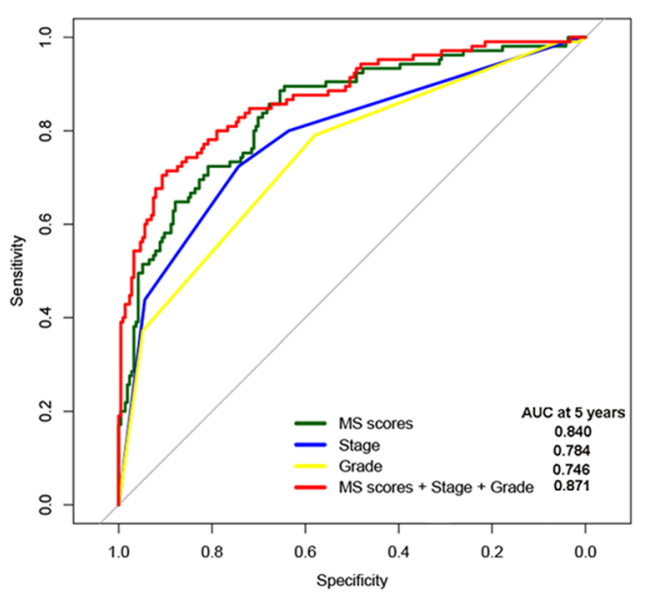

c

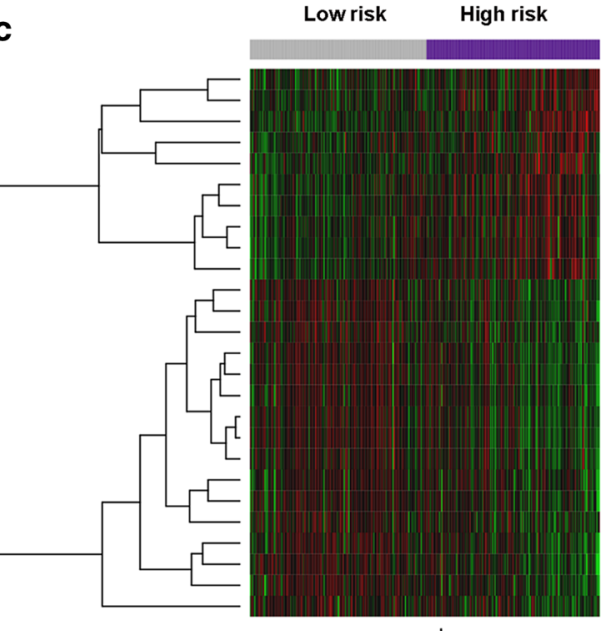

samples
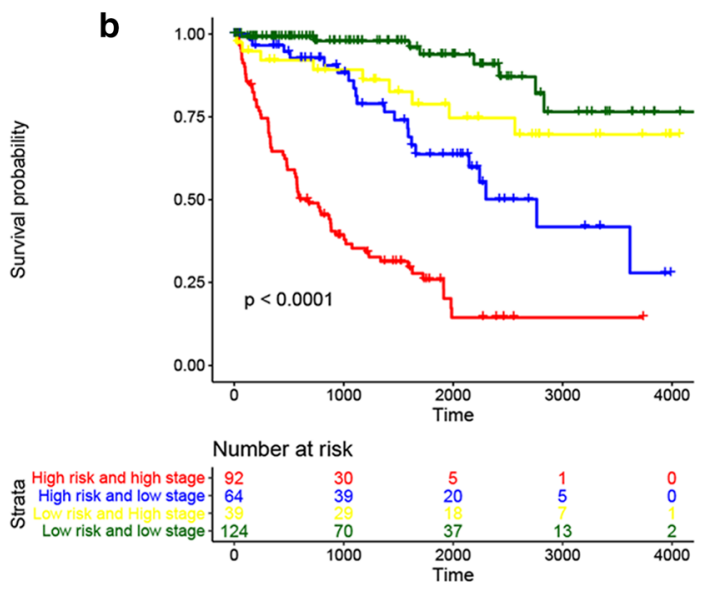

\section{d}

GLYCOSAMINOGLYCAN BIOSYNTHESIS CHON GLYCOSAMINOGLYCAN BIOSYNTHESIS KERA P53 SIGNALING PATHWAY SULFUR METABOUSM ALPHA LINOLENIC ACID METABOLISM PRIMARY IMMUNODEFICIENCY CYTOSOLIC DNA SENSING PATHWAY HEMATOPOIETIC CELL LINEAGE CYTOKINE CYTOKINE RECEPTOR INTERACT NOD LIKE RECEPTOR SIGNALING PATHWAY CITRATE CYCLE TCA CYCLE GLYCOLYSIS GLUCONEOGENESIS TERPENOID BACKBONE BIOSYNTHESIS LIMONENE AND PINENE DEGRADATION FATTY ACID METABOLISM BETA ALANINE METABOLISM VALINE LEUCINE AND ISOLEUCINE DEGRA PROPANOATE METABOLISM BUTANOATE METABOLISM ASCORBATE AND ALDARATE METABOLISM HISTIDINE METABOLISM ONE CARBON POOL BY FOLATE PPAR SIGNALING PATHWAY PROXIMAL TUBULE BICARBONATE RECLAMA RENIN ANGIOTENSIN SYSTEM
CIRCADIAN RHYTHM MAMMAL

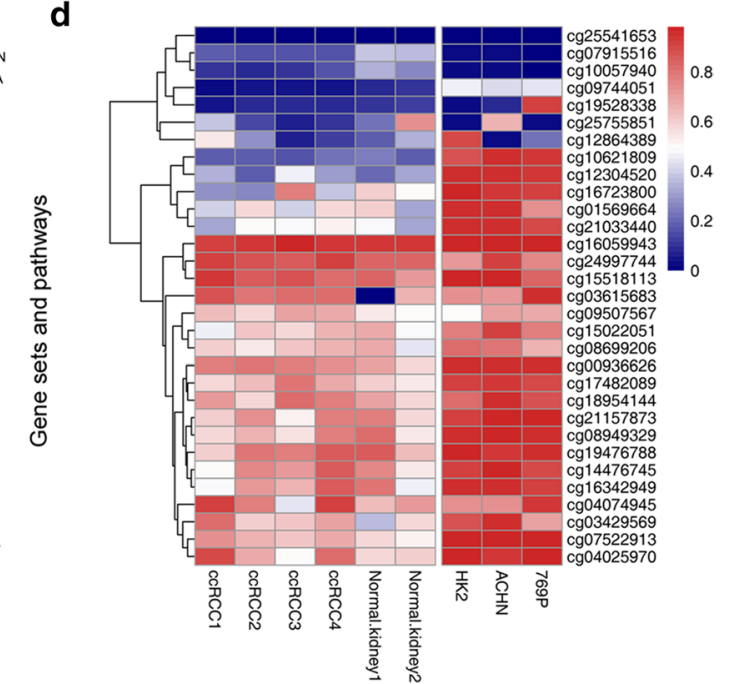

Fig. 7 ROC analysis and Subgroup analysis of the 31-CpG-based epigenetic signature. a Comparisons of the prognostic accuracy by the epigenetic signature (high risk vs low risk), histologic grade (III/IV vs I/II), pathologic stage (III/IV vs I/II) or the epigenetic signature and clinicopathological prognostic factor combined. $\mathbf{b}$ Subgroup analysis with pathologic stage of entire set. $\mathbf{c}$ The heatmap of the results of sSGSEA. The columns (represents ccRCC patients) were ordered from low to high based on epigenetic signature scores. $\mathbf{d}$ The heatmap of the $\mathrm{CpG}$ islands methylation level in the different tissues, cell lines

the prediction of the prognosis of ccRCC. And we constructed a nomogram based on this epigenetic signature, which could accurately and conveniently predict the prognosis of ccRCC patients for clinicians.

\section{Supplementary information}

Supplementary information accompanies this paper at https://doi. org/10.1186/s12935-020-01640-x.

Additional file 1. Supplementary tables 1-2, Supplementary Information: Related file 1. Ethics Committee Approval (number: 2020102).

Additional file 2. Supplementary figure S1: Functional annotation of the epigenetic signature.

Additional file 3. Supplementary figure S2: The PPI network diagram.

\section{Abbreviations}

RCC: Renal cell carcinoma; ccRCC: Clear cell renal cell carcinoma; LASSO: Least absolute shrinkage and selection operator; TCGA: The cancer genome atlas; SNP: Single nucleotide polymorphism; DMP: Differentially methylated positions; FDR: False discovery rate; PPI: Protein-protein interaction.

\section{Acknowledgements}

The excellent technical assistance of Shanshan Zhang and Danni Shan is gratefully acknowledged.

\section{Authors' contributions}

YW, XW, YX, and GW conceived and designed the study, YW and LC performed the analysis procedures, YW, LC, LJ, KY, GW, XL and YX analyzed the results, YW, LC and YX contributed analysis tools, YW, LC, XW and YX contributed to the writing of the manuscript. All authors reviewed the manuscript. All authors read and approved the final manuscript.

\section{Funding}

This work was supported by grants from the Health commission of Hubei Province scientific research project (Grant Number WJ2019H013) and the 
Fundamental Research Funds for the Central Universities (Grant Number 2042019kf0176).

\section{Availability of data and materials}

The datasets analyzed during the current study are available in the Cancer Genome Atlas (TCGA) database (http://cancergenome.nih.gov/).

\section{Ethics approval and consent to participate}

The human tissues used in the experiment were approved by the patients and approved by the Medical Ethics Committee, Zhongnan Hospital of Wuhan University. Ethics Committee Approval (number: 2020102).

\section{Consent for publication}

Not applicable.

\section{Competing interests}

The authors declare that they have no competing interests.

\section{Author details}

${ }^{1}$ Department of Urology, Zhongnan Hospital of Wuhan University, Wuhan, China. ${ }^{2}$ Department of Biological Repositories, Zhongnan Hospital of Wuhan University, Wuhan, China. ${ }^{3}$ Human Genetics Resource Preservation Center of Hubei Province, Wuhan, China. ${ }^{4}$ Human Genetics Resource Preservation Center of Wuhan University, Wuhan, China. ${ }^{5}$ Medical Research Institute, Wuhan University, Wuhan, China. ${ }^{6}$ Laboratory of Precision Medicine, Zhongnan Hospital of Wuhan University, Wuhan, China.

Received: 25 August 2019 Accepted: 2 November 2020 Published online: 23 November 2020

\section{References}

1. Hsieh JJ, Purdue MP, Signoretti S, Swanton C, Albiges L, Schmidinger M, Heng DY, Larkin J, Ficarra V. Renal cell carcinoma. Nat Rev Dis Primers. 2017;3:17009

2. Siegel RL, Miller KD, Jemal A. Cancer Statistics, 2017. CA Cancer J Clin. 2017;67(1):7-30.

3. Ljungberg B, Bensalah K, Canfield S, Dabestani S, Hofmann F, Hora M, Kuczyk MA, Lam T, Marconi L, Merseburger AS, et al. EAU guidelines on renal cell carcinoma: 2014 update. Eur Urol. 2015;67(5):913-24.

4. Esteller M. Epigenetics in cancer. N Engl J Med. 2008;358(11):1148-59.

5. Shen S, Wang G, Shi Q, Zhang R, Zhao Y, Wei Y, Chen F, Christiani DC. Seven$\mathrm{CpG}$-based prognostic signature coupled with gene expression predicts survival of oral squamous cell carcinoma. Clin Epigenet. 2017;9:88.

6. Qiu J, Peng B, Tang Y, Qian Y, Guo P, Li M, Luo J, Chen B, Tang H, Lu C, et al. $\mathrm{CpG}$ methylation signature predicts recurrence in early-stage hepatocellular carcinoma: results from a multicenter study. J Clin Oncol. 2017;35(7):734-42.

7. Garcia-Manero G, Jabbour E, Borthakur G, Faderl S, Estrov Z, Yang H, Maddipoti S, Godley LA, Gabrail N, Berdeja JG, et al. Randomized open-label phase II study of decitabine in patients with low- or intermediate-risk myelodysplastic syndromes. J Clin Oncol. 2013;31 (20):2548-53.

8. Ng SW, Mitchell A, Kennedy JA, Chen WC, McLeod J, Ibrahimova N, Arruda A, Popescu A, Gupta V, Schimmer AD, et al. A 17-gene stemness score for rapid determination of risk in acute leukaemia. Nature. 2016;540(7633):433-7.

9. Qu L, Wang ZL, Chen Q, Li YM, He HW, Hsieh JJ, Xue S, Wu ZJ, Liu B, Tang $\mathrm{H}$, et al. Prognostic value of a long non-coding RNA signature in localized clear cell renal cell carcinoma. Eur Urol. 2018;74(6):756-63.

10. Chen L, Luo Y, Wang G, Qian K, Qian G, Wu CL, Dan HC, Wang X, Xiao Y. Prognostic value of a gene signature in clear cell renal cell carcinoma. J Cell Physiol. 2019;234(7):10324-35.

11. Morris TJ, Butcher LM, Feber A, Teschendorff AE, Chakravarthy AR, Wojdacz TK, Beck S. ChAMP: 450k chip analysis methylation pipeline. Bioinformatics. 2014;30(3):428-30.

12. Teschendorff AE, Marabita F, Lechner M, Bartlett T, Tegner J, GomezCabrero D, Beck S. A beta-mixture quantile normalization method for correcting probe design bias in Illumina Infinium 450 k DNA methylation data. Bioinformatics. 2013;29(2):189-96.

13. Sandoval J, Mendez-Gonzalez J, Nadal E, Chen G, Carmona FJ, Sayols S, Moran S, Heyn H, Vizoso M, Gomez A, et al. A prognostic DNA methylation signature for stage I non-small-cell lung cancer. J Clin Oncol. 2013;31(32):4140-7.

14. Simon N, Friedman J, Hastie T, Tibshirani R. Regularization paths for Cox's proportional hazards model via coordinate descent. J Stat Softw. 2011;39(5):1-13.

15. Pencina MJ, D'Agostino RB. Overall $C$ as a measure of discrimination in survival analysis: model specific population value and confidence interval estimation. Stat Med. 2004;23(13):2109-23.

16. Szklarczyk D, Franceschini A, Wyder S, Forslund K, Heller D, Huerta-Cepas J, Simonovic M, Roth A, Santos A, Tsafou KP, et al. STRING v10: proteinprotein interaction networks, integrated over the tree of life. Nucleic Acids Res. 2015:43(Database issue):D447-52.

17. Hanzelmann S, Castelo R, Guinney J. GSVA: gene set variation analysis for microarray and RNA-seq data. BMC Bioinform. 2013;14:7.

18. Simon R, Altman DG. Statistical aspects of prognostic factor studies in oncology. Br J Cancer. 1994;69(6):979-85.

19. Goeman JJ. L1 penalized estimation in the Cox proportional hazards model. Biom J. 2010;52(1):70-84.

20. Zhang JX, Song W, Chen ZH, Wei JH, Liao YJ, Lei J, Hu M, Chen GZ, Liao $B$, Lu J, et al. Prognostic and predictive value of a microRNA signature in stage II colon cancer: a microRNA expression analysis. Lancet Oncol. 2013;14(13):1295-306.

21. Jiang Y, Zhang Q, Hu Y, Li T, Yu J, Zhao L, Ye G, Deng H, Mou T, Cai S, et al. ImmunoScore signature: a prognostic and predictive tool in gastric cancer. Ann Surg. 2018;267(3):504-13.

22. Tian XP, Huang WJ, Huang HQ, Liu YH, Wang L, Zhang X, Lin TY, Rao $\mathrm{HL}$, Li M, Liu F, et al. Prognostic and predictive value of a microRNA signature in adults with T-cell lymphoblastic lymphoma. Leukemia. 2019;33(10):2454-65.

23. Luo Y, Chen L, Wang G, Xiao Y, Ju L, Wang X. Identification of a threemiRNA signature as a novel potential prognostic biomarker in patients with clear cell renal cell carcinoma. J Cell Biochem. 2019;120(8):13751-64.

24. Rondinelli B, Rosano D, Antonini E, Frenquelli M, Montanini L, Huang D, Segalla S, Yoshihara K, Amin SB, Lazarevic D, et al. Histone demethylase JARID1C inactivation triggers genomic instability in sporadic renal cancer. J Clin Invest. 2015;125(12):4625-37.

25. Li Y, Gong Y, Ning X, Peng D, Liu L, He S, Gong K, Zhang C, Li X, Zhou L. Downregulation of CLDN7 due to promoter hypermethylation is associated with human clear cell renal cell carcinoma progression and poor prognosis. J Exp Clin Cancer Res. 2018;37(1):276.

26. Gooskens SL, Klasson TD, Gremmels H, Logister I, Pieters R, Perlman EJ, Giles RH, van den Heuvel-Eibrink MM. TCF21 hypermethylation regulates renal tumor cell clonogenic proliferation and migration. Mol Oncol. 2018;12(2):166-79.

27. Geybels MS, Wright JL, Bibikova M, Klotzle B, Fan JB, Zhao S, Feng Z, Ostrander EA, Lin DW, Nelson PS, et al. Epigenetic signature of Gleason score and prostate cancer recurrence after radical prostatectomy. Clin Epigenet. 2016;8:97.

28. Wang X, Cheng Y, Zhu Y, Li H, Ge W, Wu X, Zhao K, Yuan J, Li Z, Jiang $\mathrm{S}$, et al. Epigenetic silencing of ASPP1 confers 5-FU resistance in clear cell renal cell carcinoma by preventing p53 activation. Int J Cancer. 2017;141(7):1422-33.

29. Mastropasqua F, Marzano F, Valletti A, Aiello I, Di Tullio G, Morgano A, Liuni S, Ranieri E, Guerrini L, Gasparre G, et al. TRIM8 restores p53 tumour suppressor function by blunting N-MYC activity in chemo-resistant tumours. Mol Cancer. 2017;16(1):67.

30. Mey L, Jung M, Roos F, Blaheta R, Hegele A, Kinscherf R, Urbschat A. NOD1 and NOD2 of the innate immune system is differently expressed in human clear cell renal cell carcinoma, corresponding healthy renal tissue, its vasculature and primary isolated renal tubular epithelial cells. J Cancer Res Clin Oncol. 2019;145(6):1405-16.

31. Du W, Zhang L, Brett-Morris A, Aguila B, Kerner J, Hoppel CL, Puchowicz M, Serra D, Herrero L, Rini BI, et al. HIF drives lipid deposition and cancer in cCRCC via repression of fatty acid metabolism. Nat Commun. 2017;8(1):1769. 
32. Wettersten HI, Aboud OA, Lara PN Jr, Weiss RH. Metabolic reprogramming in clear cell renal cell carcinoma. Nat Rev Nephrol. 2017;13(7):410-9.

33. Theoleyre $\mathrm{S}$, Mottier S, Masson D, Denis MG. HtrA3 is regulated by 15-deoxy-Delta 12,14-prostaglandin J2 independently of PPARgamma in clear cell renal cell carcinomas. Biochem Biophys Res Commun. 2010;394(3):453-8.

\section{Publisher's Note}

Springer Nature remains neutral with regard to jurisdictional claims in published maps and institutional affiliations.
Ready to submit your research? Choose BMC and benefit from:

- fast, convenient online submission

- thorough peer review by experienced researchers in your field

- rapid publication on acceptance

- support for research data, including large and complex data types

- gold Open Access which fosters wider collaboration and increased citations

- maximum visibility for your research: over $100 \mathrm{M}$ website views per year

At BMC, research is always in progress.

Learn more biomedcentral.com/submissions 